beiter regeln lassen. Auch diese Delegation von Kommunikation, mit der die Responsivitätsrolle in gewissem Maße verlagert wird, ist bei der weiteren Diskussion über die Demokratisierungspotenziale des Internets für die politische Kommunikation zu beachten.

Allerdings werden die skizzierten Dysfunktionen insbesondere von jenen Abgeordneten gesehen, die sich als Online-Überforderte und Online-Skeptiker herausstellen. Dabei handelt es sich vornehmlich um ältere $\mathrm{MdBs}$ mit vergleichsweise geringer Internetkompetenz. Ihnen gegenüber stehen die Online-Souveränen und funktionalen Online-Nutzer, die, mit hoher Internetkompetenz ausgestattet, in stärkerem Maße die Vorteile der Online-Kommunikation zur (Re-)Präsentation und für ihre politische Arbeit sehen und die auch überdurchschnittlich häufig selbst in den E-Mail-Austausch mit den Bürgern eintreten. Diese beiden Gruppen stellen schon jetzt etwas mehr als die Hälfte der Parlamentarier.

Diese Befunde deuten darauf hin, dass es derzeit eine Art Digital Divide quer durch die parlamentarischen Reihen gibt. Diese trennt die internetaffineren jüngeren Abgeordneten von den internetzögerlicheren älteren. Es ist jedoch zu erwarten, dass sich in den kommenden Jahren mit dem Voranschreiten der jüngeren Parlamentarierkohorten und der immer alltäglicher werdenden digitalen Kommunikationsmöglichkeiten der Stellenwert der Online-Kommunikation ändern wird: weg von einer (noch) symbolischen hin zu einer stärker funktionalen Nutzung. Die schon jetzt hohe Internetaktivität der Abgeordneten könnte so in noch höherem Maße Angebote zur Interaktivität schaffen. Inwieweit diese von den Bürgern dann auch genutzt werden, inwieweit sich dadurch das Repräsentationsverhältnis und letztlich die parlamentarische Arbeit verändern, wird weiter zu beobachten sein.

\title{
Mandatstypen und Ausschussmitgliedschaften der Mitglieder des Deutschen Bundestags - Eine empirische Untersuchung von 1949 bis $2005^{*}$
}

\section{Dominic Heinz}

Im Deutschen Bundestag ist die Mitgliedschaft in Ausschüssen von großer Bedeutung, denn die Abgeordneten wirken dadurch an der Gesetzgebung mit, und Ausschüsse verbinden Abgeordnete, Minister, Ministerialverwaltung und Interessenvertreter. Dabei sind die Parlamentarier in den Ausschüssen weder reine Agenten ihrer Fraktionen, noch können sie sich ihren Ausschuss frei aussuchen. Relevante politikwissenschaftliche Forschung widmete sich der Frage, wie Abgeordnete ihre Ausschussmitgliedschaft erlangen. Nachdem die Ausschussstruktur des Bundestages feststeht und die auf die einzelnen Fraktionen entfallende

* Ausführlichere Versionen des Beitrags konnten am Brown Bag Kolloquium des Mannheimer Zentrums für Europäische Sozialforschung (MZES), auf der 2. ECPR Graduiertenkonferenz, an der DVPW Sektionstagung „Vergleichende Politikwissenschaft“ und auf dem 105. APSA Annual Meeting 2009 vorgestellt werden. Allen Teilnehmern und der Redaktion der Zeitschrift für Parlamentsfragen bin ich für ihre Anmerkungen zu Dank verpflichtet. Darüber hinaus danke ich Philip Manow, Bernhard Miller, Thomas Saalfeld, Arndt Wonka, Andreas M. Wïst und Thomas Zittel. 
Anzahl von Ausschusssitzen ermittelt worden ist ${ }^{1}$, koordinieren Parlamentarische Geschäftsführer als Teil der Fraktionsführung die Zuteilung der Sitze an die Abgeordneten. ${ }^{2}$ Während das Ergebnis formell von der Vollversammlung der Fraktion beschlossen wird, ist die inhaltliche Entscheidung Ergebnis von Verhandlungen zwischen der Fraktionsführung und den Abgeordneten, bei dem Angebot und Nachfrage von Ausschusssitzen so gut wie möglich in Übereinstimmung gebracht werden müssen. ${ }^{3}$ Dabei verfolgen Fraktionen zunächst allgemeine Gründe, wie Sachverstand in den Gesetzgebungsprozess einzubinden oder eine einheitliche Fraktionslinie zu verfolgen. Darüber hinaus ergeben sich je nach Ausschuss unterschiedliche Auffälligkeiten, bei denen persönliche Merkmale der Abgeordneten in Verbindung mit der inhaltlichen Ausrichtung des Ausschusses zu stehen scheinen. So befinden sich im Auswärtigen Ausschuss meistens erfahrene Politiker mit hoher Reputation. Neu gewählte Abgeordnete sind oft Mitglied im Petitionsausschuss. ${ }^{4}$ MdBs, die eine juristische Ausbildung haben oder eine anwaltliche Tätigkeit verfolgen, frequentieren den Rechtsausschuss; Angestellte in Gewerkschaften und Arbeitnehmerorganisationen sind häufig Mitglied im Ausschuss für Arbeit und Sozialordnung. ${ }^{5}$ Abgeordnete mit Migrationshintergrund sind vielfach in Ausschüssen mit migrationsnahen Themen zu finden. ${ }^{6}$ Außer den persönlichen Merkmalen Seniorität, Ausbildung, Beruf und Herkunft erscheint die lokale Färbung der Abgeordneten ebenso wichtig für die Ausschussmitgliedschaft. ${ }^{7}$

\section{Eine Verbindung von Mandatstyp und Ausschussmitgliedschaft?}

Öffentliche Medienberichterstattung erkennt aber auch vereinzelt einen Zusammenhang zwischen der Mitgliedschaft in besonderen Ausschüssen und dem Mandatstyp von Bundestagsabgeordneten. Zum Beispiel verbreitet die im Landwirtschaftsausschuss tätige Direktabgeordnete Julia Klöckner (CDU) in der Lokalpresse, wie viel Geld von der Bundesregierung in Projekte in ihrem Wahlkreis geflossen ist. ${ }^{8}$ Ein anderes Beispiel zeigte sich, als Oswald Metzger versuchte, von der CDU als Direktkandidat nominiert zu werden, da sich der Wahlkreis „von dem bisherigen Bundestagsabgeordneten Andreas Schockenhoff (CDU) schlecht vertreten [fühlte], weil Bundesstraßen noch immer nicht ausgebaut [wurden] "9). Eine Verbindung zwischen Wahlkreisinteressen und Ausschussarbeit von Bundestagsabgeordneten

1 Vgl. Susanne Linn / Frank Sobolewski, So arbeitet der Deutsche Bundestag - Organisation und Arbeitsweise. Die Gesetzgebung des Bundes, Rheinbreitbach 2010, S. 20.

2 Vgl. Suzanne S. Schüttemeyer, Fraktionen im Deutschen Bundestag 1949-1997. Empirische Befunde und theoretische Überlegungen, Opladen 1998, S. 278 - 285.

3 Vgl. Wolfgang Ismayr, Der Deutsche Bundestag, Opladen 2000, S. $171 \mathrm{ff}$.

4 Vgl. ebenda, S. 382.

5 Vgl. Peter Schindler, Datenhandbuch zur Geschichte des Deutschen Bundestages 1949 bis 1999, Band II, Baden-Baden 1999, S. 2113.

6 Vgl. Andreas M. Wüst / Dominic Heinz, Die politische Repräsentation von Migranten in Deutschland, in: Markus Linden / Winfried Thaa (Hrsg.), Die politische Repräsentation von Fremden und Armen, Baden-Baden 2009, S. $201-218$.

7 Vgl. Thomas Gschwend / Matthew S. Shugart / Thomas Zittel, Assigning Committee Seats in Mixed-Member-Systems - How Important is „Localness“ Compared to the Mode of Election?, Papier für die ECPR General Conference vom 8. bis 10. September 2009 in Potsdam.

8 Vgl. Nahe-Zeitung, Geld für Campus - Fördermittel verteilt, 7. Juli 2008.

9 Rüdiger Soldt, Wie halten Sie es mit der Selbstdarstellung?, FAZ.NET vom 20. September 2008. 
wurde auch bei der verspäteten Einführung des LKW-Mautsystems vermutet. Dies hätte zu Einnahmeausfällen führen können, so dass deswegen der Bundestagsabgeordnete im Verkehrsausschuss Dirk Fischer (CDU) „befürchtete, jetzt könnten Verkehrsprojekte gestrichen werden. Vielleicht trifft es auch sein Lieblingsprojekt: die A6 in seinem Wahlkreis"10.

Darüber hinaus finden sich in politikwissenschaftlichen Fachzeitschriften Annahmen, dass Wahlkreisprojekte für Direktabgeordnete einen höheren Stellenwert besitzen als für Listenabgeordnete. ${ }^{11}$ In einer anderen Studie wird argumentiert, dass sich die Ausschüsse im Bundestag eher in wahlkreisorientierte (Landwirtschaft, Verkehr und Bau) und parteiorientierte (Gesundheit, Verteidigung, Familie und Entwicklung) einteilen lassen. ${ }^{12} \mathrm{Nimmt}$ man den Zeitraum von 1990 bis 2002 zur Referenz, scheinen Wahlkreisabgeordnete andere Ausschüsse als Listenabgeordnete zu frequentieren. Bestätigt sich dieser Befund bei einer Langzeituntersuchung von 1949 bis 2005? Wenn ja, wie ist der Zusammenhang zwischen Mandat und Ausschussmitgliedschaft zu erklären?

\section{Mandatstypen im Spiegel politikwissenschaftlicher Literatur}

Nach Dieter Nohlen gibt es entweder Mehrheitswahlsysteme oder Verhältniswahlsysteme. Allenfalls erfüllen einige Formen die Idealtypen nicht voll und ganz beziehungsweise sind näher oder weiter weg von den beiden Idealtypen. ${ }^{13}$ Dementsprechend betrachtete die politikwissenschaftliche Forschung zunächst traditionelle Mehrheits- oder Verhältniswahlsysteme und deren Einfluss auf die Sitzverteilung für Parteien. Zahlreiche Arbeiten befassen sich mit der Anzahl von Parteien und der Repräsentativität, die ein Wahlsystem hervorbringt und erkennen die genannten Mischwahlsysteme nicht als eigenen Systemtypus an. ${ }^{14}$ Es gibt aus dieser Sichtweise keine Mischtypen, sondern Wahlsysteme mit zwei Funktionslogiken, die jedoch keine unterschiedlichen Abgeordnetentypen produzieren. Aus diesem Grund können Listenabgeordnete im Prinzip als „verhinderte Wahlkreisabgeordnete“ 15 bezeichnet werden. Parlamentarier, die ihr Mandat über die Landesliste erlangten, unterscheiden sich nicht von Wahlkreisabgeordneten, weil sie motiviert sind, bei der nächsten Wahl das Direktmandat zu erringen. Als Begründung wird darauf verwiesen, dass Bundestagsabgeordnete in der Regel sowohl in einem Wahlkreis als auch auf einer Landesliste kandidieren. So hatten etwa im 14. und 15. Bundestag 94 Prozent der über Parteilisten ins Parlament eingezogenen MdBs auch eine Nominierung für einen Wahlkreis, und in der Tat konnte festgestellt werden, dass die Voraussetzung für einen aussichtsreichen Listenplatz die

10 Kontraste, Das Desaster mit der Maut - wer soll das bezahlen?, 25. September 2003.

11 Vgl. Thomas D. Lancaster / David W. Patterson, Comparative Pork Barrel Politics. Perceptions from the West German Bundestag, in: Comparative Political Studies, 22. Jg. (1990), H. 4, S. 458 - 477.

12 Vgl. Thomas Stratmann / Martin Baur, Plurality Rule, Proportional Representation, and the German Bundestag: How Incentives to Pork-Barrel Differ Across Electoral Systems, in: American Journal of Political Science, 46. Jg. (2002), H. 3, S. $506-514$.

13 Vgl. Dieter Nohlen, Wahlrecht und Parteiensystem: Über die politischen Auswirkungen von Wahlsystemen, Opladen 1990.

14 Vgl. Maurice Duverger, Political Parties: Their Organization and Activity in the Modern State, New York 1954; Arend Lijphart, Electoral Systems and Party Systems in Twenty-Seven Democracies, Oxford 1994.

15 Hans-Helmut Röhring / Kurt Sontheimer, Handbuch des deutschen Parlamentarismus, München 1970, S. 275. 
Kandidatur in einem Wahlkreis ist. ${ }^{16}$ Die Empirie liefert also keine Evidenz für eine Unterscheidung in zwei unterschiedliche Typen von Abgeordneten. ${ }^{17}$

Durch die Wahlkandidatur ihrer Listenabgeordneten gewinnen vor allem kleine Parteien, die so gut wie nie ein Direktmandat erringen, ihre Zweitstimme. ${ }^{18}$ Dass die zusätzliche Direktkandidatur eher auf die Zweitstimme ausgerichtet ist, wird durch den - plausiblen, wenngleich nur für die Bundestagswahl 2002 systematisch erhärteten - Eindruck verstärkt, dass Direktkandidaturen in den kleinen Parteien selten umstritten sind. ${ }^{19}$ Auch aus diesem Grund hat der Mandatstyp eines Abgeordneten im Bundestag keine besondere Bedeutung. "Contrary to widespread opinion, it is of absolutely no importance whether a mandate is obtained through the constituency or the Landesliste." ${ }^{20}$ Diese Sichtweise der 1980er Jahre ist auch weiterhin im Kanon aktueller Lehrbücher zu finden: „Zu erwähnen ist im übrigen, dass es in der Praxis kaum einen Unterschied macht, ob ein Abgeordneter sein Mandat direkt oder über die Landesliste erhalten hat." 21

\section{Direkt- und Listenmandate von 1949 bis 2005}

Für jeden Abgeordneten ist der Mandatstyp eindeutig, weil Mitglieder des Bundestages nur über eine der beiden Wahlregeln gewählt werden können. Aus den biographischen Angaben der MdBs sind die Informationen zu entnehmen, in welchem Ausschuss der jeweilige Abgeordnete war, über welche Wahlregel er gewählt wurde und wo er zusätzlich kandidiert hat. ${ }^{22}$ Die Angaben aus der Sekundärliteratur wurden für Abgeordnete jüngerer Wahlperioden durch offen zugängliche Quellen wie Parlamentshandbücher und Internetseiten vervollständigt.

Von 1949 (Anfang 1. Wahlperiode) bis 2005 (Ende 15. Wahlperiode) wurden 3.193 Bundestagsabgeordnete erfasst. Die Literatur berichtet von 3.178 Abgeordneten zu Beginn der 15. Wahlperiode. ${ }^{23}$ Dazu sind die Mandatsänderungen während der 15. Wahlperiode zu addieren. ${ }^{24}$ Es müssten sich 3.195 Abgeordnete ergeben, so dass zwei Abgeordnete oder 0,06 Prozent in der diesem Beitrag zu Grunde gelegten Datenbank fehlen.

16 Vgl. Suzanne S. Schüttemeyer / Roland Sturm, Der Kandidat - das (fast) unbekannte Wesen: Befunde und Überlegungen zur Aufstellung der Bewerber zum Deutschen Bundestag, in: ZParl, 36. Jg. (2005), H. 3, S. 539 - 553, S. $547 \mathrm{ff}$.

17 Vgl. Eckhard Jesse, Split-voting in the Federal Republic of Germany: An Analysis of the Federal Elections from 1953 to 1987, in: Electoral Studies, 7. Jg. (1988), H. 2, S. $109-124$.

$18 \mathrm{Vgl}$. Karen E. Cox / Leonard J. Schoppa, Interaction Effects in Mixed-Member Electoral Systems: Theory and Evidence From Germany, Japan, and Italy, in: Comparative Political Studies, 35. Jg. (2002), H. 9, S. 1027 - 1053.

19 Vgl. Suzanne S. Schüttemeyer / Roland Sturm, a.a.O (Fn. 16).

20 Tony Burkett / Stephen Padgett, Political Parties and Elections in West Germany. The Search for a New Stability, London 1987, S. 130.

21 Dieter Nohlen, Wahlrecht und Parteiensystem. Zur Theorie der Wahlsysteme, Stuttgart 2003, S. 307.

22 Vgl. Rudolf Vierhaus / Ludolf Herbst, Biographisches Handbuch der Mitglieder des Deutschen Bundestages 1949-2002, München 2002; Michael F. Feldkamp, Datenhandbuch zur Geschichte des Deutschen Bundestages 1994 bis 2003, Berlin 2004; Peter Schindler, a.a.O. (Fn. 5).

23 Vgl. Michael F. Feldkamp, a.a.O. (Fn. 22), S. 115.

24 Vgl. Kürschners Volkshandbuch, Deutscher Bundestag - 16. Wahlperiode, Bad Honnef 2006, S. 267. 
Die Gesamtzahl der Mandate von 1949 bis 2005 beträgt 8.925. Davon sind 3.990 (oder 44,7 Prozent) Direktmandate, und 4.935 (oder 55,3 Prozent) entfallen auf die Landeslisten der Parteien. Hinsichtlich der gesamten Mandatszahl (mit Berliner und saarländischen Abgeordneten, ohne DDR-Volkskammerdelegierte) ist festzuhalten, dass es 17 von 8.925 oder 0,2 Prozent Mandate gibt, die in der verwendeten Datenbank falsch zugeordnet wurden.

\begin{tabular}{|l|c|c|}
\hline Tabelle 1: Direkt- und Listenmandate im Deutschen Bundestag 1949 bis 2005 \\
\hline Typ des Bundestagsmandats & $\mathrm{N}$ & Prozent \\
\hline Direktmandat & 3.990 & 44,7 \\
Listenmandat & 4.935 & 55,3 \\
Total & 8.925 & 100,0 \\
\hline Quelle: Eigene Berechnungen. \\
\hline
\end{tabular}

Das Verhältnis von Direkt- zu Listenabgeordneten ist wegen der Nachrücker nicht $50 \mathrm{zu}$ 50, wie man aus den Wahlregeln vermuten könnte, sondern leicht zu Gunsten von Listenabgeordneten verschoben. Tabelle 1 berichtet die relativen und absoluten Häufigkeiten des Mandatstyps. Das Mischwahlsystem beziehungsweise das Wahlsystem mit zwei Funktionslogiken und die zusätzliche Absicherung von Abgeordneten auf der Liste oder über einen Wahlkreis haben allerdings zur Folge, dass sich theoretisch der Mandatstyp von einer Wahl zur nächsten ändern kann. Je nach dem, ob es der Direktkandidat schafft, das Wahlkreismandat zu erringen, oder ob der Direktabgeordnete sein Direktmandat verliert und durch einen sicheren Listenplatz trotzdem in den Bundestag einzieht, kann sich der Mandatstyp bei Bundestagsabgeordneten auch verändern.

In der Zeit von 1949 bis 2005 sind 314 Direktabgeordnete oder etwa vier Prozent in der folgenden Wahlperiode mit einem Listenmandat wiedergewählt worden. Umgekehrt haben 414 Listenabgeordnete oder etwas über fünf Prozent ein Direktmandat bei der folgenden Wahl erlangt. Eine Änderung des Mandatstyps ist demnach eine Ausnahme. Dieser Befund erhärtet sich vor dem Hintergrund, dass Abgeordnete kleiner Parteien fast nur über ihre Parteilisten in den Bundestag einziehen können. Ausnahmen finden sich bei der Partei Die Linke in der Bundestagswahl 2009, in den Anfangsjahren der Bundesrepublik und in der Zeit nach der Wiedervereinigung bei der PDS.

Karrieren wie zum Beispiel die von Volker Hauff(SPD) stellen eine Seltenheit dar. Hauff war von der 6. bis zur 11. Wahlperiode Bundestagsabgeordneter. In der 6. Wahlperiode wurde er über die Landesliste Baden-Württemberg gewählt, in der 7. Wahlperiode wurde er im Wahlkreis 171 direkt gewählt, in der 8. Wahlperiode setzte er seine Abgeordnetenkarriere erneut über das Mandat der Landesliste Baden-Württemberg fort, um in der 9. Wahlperiode wieder direkt im Wahlkreis 165 gewählt zu werden und anschließend in der 10. und 11. Wahlperiode noch einmal über die Liste in den Bundestag einzuziehen. Ebenso wechselte zum Beispiel der Abgeordnete Herbert Hauffe (SPD) zwischen Wahlkreismandat in der 2. und 4. Wahlperiode und dem Listenmandat für den 3. und 5. Bundestag. Diese Muster betreffen allerdings nur unter zehn Prozent der Mandate, so dass der Abgeordnete im Normalfall entweder seine oder ihre legislative Karriere über das Wahlkreis- oder das Listenmandat bestreitet, so wie es etwa beim Abgeordneten Wolfgang Mischnick (FDP) der Fall war. Er erlangte sämtliche zehn Mandate über die FDP-Liste. Auch zum Beispiel die Karriere von Albert Probst (CSU) spiegelt eher den Normalfall wider: Er wurde von der 6. bis zur 13. Wahlperiode über einen Wahlkreis in den Bundestag entsandt. 


\section{Ausschüsse im Bundestag}

Der Bundestag wird im Allgemeinen als Arbeitsparlament klassifiziert, weil die Hauptarbeit für seine Mitglieder in den Ausschüssen stattfindet. Wenn die Abgeordneten nicht in ihren Wahlkreisen tätig sind, gilt der Ausschussarbeit die größte Aufmerksamkeit in den Sitzungswochen. Artikel 45 des Grundgesetzes verlangt die Einsetzung der Ausschüsse für Auswärtiges, für Verteidigung, Petitionen und Angelegenheiten der EU. Der Haushaltsausschuss sowie der Ausschuss für Wahlprüfung, Immunität und Geschäftsordnung müssen vom Bundestag aufgrund einfachgesetzlicher Regelungen eingesetzt werden. Darüber hinaus ist der Bundestag frei in seiner Ausschusswahl. ${ }^{25}$ Einige Ausschüsse existierten nur ein einziges Mal, zum Beispiel der Olympia-Ausschuss 1969, andere wurden über mehrere Wahlperioden eingesetzt. Insgesamt lassen sich zwölf Ausschüsse erkennen, die in jeder Wahlperiode von 1949 bis 2005 eingerichtet wurden, von denen Stratmann und Baur sieben in Wahlkreis- und Parteiausschüsse einteilen. Die Ausschüsse für Inneres, Wirtschaft, Finanzen, Recht und Soziales konnten sie nicht eindeutig zuordnen.

Nach dem Argument von Stratmann und Baur eignen sich der Verkehrs-, Landwirtschafts- und Bauausschuss besonders für die Repräsentation von Wahlkreisinteressen. Am deutlichsten wird dies im Verkehrsausschuss. Dort werden seit 1973 Bundesverkehrswegepläne beraten, die mit dem Ausbau von Schienen, Straßen und Wasserstraßen Investitionsrahmen und Steuerungsinstrument der Bundesregierung darstellen. Für Ausschussmitglieder ergeben sich daher Anreize, Ressourcen besonders für ihren Wahlkreis zu mobilisieren. Obwohl der Landwirtschaftsausschuss seit neuestem als Verbraucherschutzausschuss Karriere gemacht hat, beschäftigt er sich nach wie vor hauptsächlich mit Angelegenheiten der Landwirte. Rechtliche Regelungen zum Beispiel zur Tierhaltung und zum Status geographischer Flächen passieren trotz der Gemeinsamen Agrarpolitik (GAP) der Europäischen Union (EU) den Landwirtschaftsausschuss und sind für die Interessen im ländlichen Raum von zentraler Bedeutung. Gleiches trifft für den Bauausschuss zu, in dem vor allem in der Nachkriegszeit über Wohnungsbauprogramme beraten und der Wiederaufbau mit gesteuert wurde. Auch die Angelegenheiten des Wohnungsbaus waren beziehungsweise sind für Wahlkreisinteressen relevant.

Als Parteiausschüsse, die sich laut Stratmann und Baur zur Repräsentation parteipolitischer Programmatik eignen, sind der Entwicklungs-, Familien-, Gesundheits- und Verteidigungsausschuss zu nennen. Letztere diente in der Zeit von 1949 bis 2005 hauptsächlich zum Aufbau und danach zur parlamentarischen Kontrolle der Bundeswehr. Der Ausschuss gibt außerdem Empfehlungen an die Regierung zur Sicherheits- und Verteidigungspolitik. Dass Direktabgeordnete zum Beispiel durch Rüstungsunternehmen in ihrem Wahlkreis möglicherweise motiviert werden, Mitglied im Verteidigungsausschuss zu werden, kann nicht ausgeschlossen werden. In dem langen Zeitraum von 1949 bis 2005 ist dies allerdings nicht das zentrale Arbeitsfeld des Ausschusses. Dort ging es und geht es weiterhin tatsächlich um parlamentarische Kontrolle der Bundeswehr sowie sicherheits- und verteidigungspolitische Empfehlungen, die kaum mit materiellen Ressourcen für Wahlkreise verbunden sind. Ähnliche Beratungsfunktionen führen auch der Ausschuss für Gesundheit, für Familie und für Entwicklung aus, ohne dass dies mit einem materiellen Nutzen verbunden wäre.

25 Eine vollständige Liste aller 65 Parlamentsausschüsse von 1949 bis 2005 findet sich bei Michael F. Feldkamp, a.a.O. (Fn. 22); Peter Schindler, a.a.O. (Fn. 5). 


\begin{tabular}{|l|r|c|}
\hline Tabelle 2: Anzahl und Anteil von Wahlkreis- und Parteiausschussmitgliedschaften \\
\hline & $\mathrm{N}$ & Prozent \\
\hline Parteiausschüsse & 1.612 & 18,1 \\
Familie & 530 & \\
Gesundheit & 262 & \\
Entwicklung & 329 & \\
Verteidigung & 491 & \\
\hline Wahlkreisausschüsse & 1.388 & 15,6 \\
Landwirtschaft & 502 & \\
Verkehr & 501 & \\
Bau & 385 & \\
\hline Neutrale Ausschüsse & 5.925 & 66,3 \\
\hline Total & 8.925 & 100,0 \\
\hline Quelle: Eigene Berechnungen. & & \\
\hline
\end{tabular}

Vergegenwärtigt man sich die Themen wie die in der öffentlichen Diskussion so genannte Herdprämie, Kopfpauschale oder Bürgerversicherung, kommt damit eine bestimmte parteipolitische Programmatik zum Ausdruck und keine Wahlkreisinteressen.

Von den insgesamt 8.925 Abgeordneten, die in einem Bundestagsausschuss Mitglied waren, entfielen 1.388 (oder 15,6 Prozent) auf Wahlkreisausschüsse und 1.612 (oder 18,1 Prozent) auf Parteiausschüsse. Zwei Drittel der Mandate waren in neutralen Ausschüssen zu verzeichnen, die weder eindeutig als Wahlkreis-, noch als Parteiausschuss klassifiziert werden können.

Unter den 1.388 Wahlkreisausschüssen fällt der Wert für den Bauausschuss deutlich geringer aus als der für die anderen Ausschüsse, weil in den letzten Wahlperioden die Ausschüsse für Bau und Verkehr zusammengefasst wurden. Dafür war der Bauausschuss vor allem in der unmittelbaren Nachkriegszeit umso wichtiger, als es um den Wiederaufbau von Wohnraum ging.

Insgesamt wechseln die Bundestagsabgeordneten zwischen Wahlkreis- und Parteiausschüssen mit Anteilen von unter einem Prozent äußerst selten. Das entspricht den empirischen Befunden der Parlamentarismusforschung, nach denen die meisten Parlamentarier Fachpolitiker sind. Innerhalb ihres Themengebiets sind sie Experten und bringen diese Expertise in die Parlaments- und Fraktionsarbeit ein. ${ }^{26}$

\section{Mandate und Ausschusstypen im Gesamtüberblick}

Die beiden vorstehenden Abschnitte zeigten im Mandatstyp und in der Ausschussmitgliedschaft große Stabilität. Hinsichtlich ihres in Medienberichten und politikwissenschaftlichen Studien behaupteten Zusammenhanges gibt Tabelle 3 einigen Aufschluss.

26 Vgl. ebenda. 


\begin{tabular}{|l|c|}
\hline Tabelle 3: Prozentuale Anteile von Direktmandaten in Ausschusstypen \\
\hline Anteile Direktmandate von & \\
\hline allen Mandaten von 1949 bis 2005 & 44 \\
allen Mandaten in Parteiausschüssen & 39 \\
allen Mandaten in Wahlkreisausschüssen & 53 \\
allen Mandaten in neutralen Ausschüssen & 44 \\
\hline Quelle: Eigene Berechnungen & \\
\hline
\end{tabular}

Zwischen 1949 und 2005 hatten 44 Prozent aller Bundestagsabgeordneter ein Direktmandat inne. In Wahlkreisausschüssen waren mit 53 Prozent mehr direkt gewählte Abgeordnete vertreten als in Parteiausschüssen. Im Gegensatz dazu waren nur 39 Prozent Direktabgeordnete Mitglied in Parteiausschüssen. In neutralen Ausschüssen waren mit 44 Prozent durchschnittlich viele Direktabgeordnete tätig. In 121 Fällen war der jeweilige Abgeordnete sowohl in einem Wahlkreis- als auch in einem Parteiausschuss Mitglied. Angesichts der geringen Fallzahl (121 im Vergleich zu 1.612 und 1.388) überrascht es nicht, dass diese nur geringe Auswirkungen auf die Ergebnisse haben. Abbildung 1 zeigt, wie sich das Verhältnis zwischen Wahlkreis- und Listenabgeordneten in Wahlkreisausschüssen über 15 Wahlperioden verändert hat.

Die große Diskrepanz zwischen Direkt- und Listenmandaten in der ersten Wahlperiode kann durch das 1949 geltende Wahlrecht erklärt werden, das 60 Prozent Direktmandate und nur 40 Prozent Listenmandate vorsah. Im dritten Bundestag gab es in Wahlkreisausschüssen mehr Abgeordnete mit Listenmandaten als mit Direktmandaten. Nach der definitiven Etablierung des heutigen Wahlsystems stellte sich der erwartete Unterschied in Wahlkreisausschüssen ein. Lediglich in der neunten und zehnten Wahlperiode sind Direkt- und Listenmandate in Wahlkreisausschüssen gleich verteilt. Komplementär dazu zeigt Abbildung 2 die Anteile von Direkt- und Listenmandatsinhabern in Parteiausschüssen.

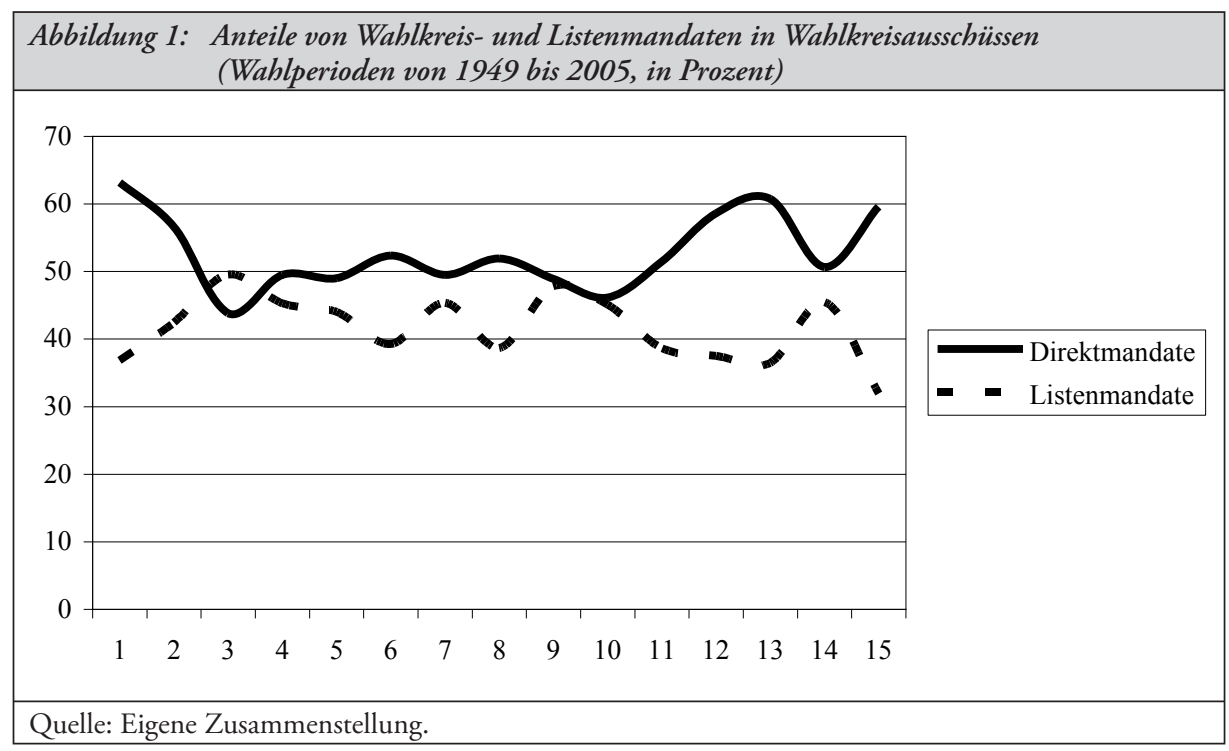




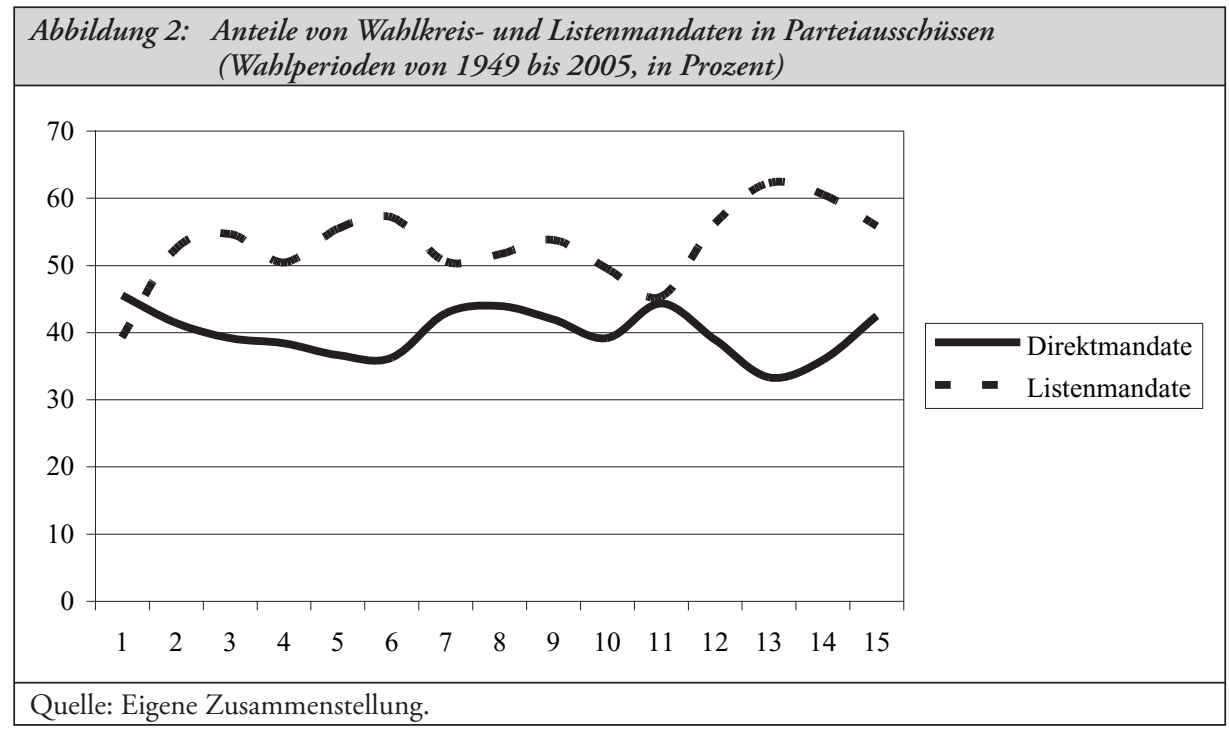

Auch hier zeigt sich in der ersten Wahlperiode ein ungewöhnliches Ergebnis: 1949 sind in den Parteiausschüssen mehr Direkt- als Listenmandatsinhaber. In der 11. Wahlperiode sind dann Direkt- und Listenabgeordnete gleichmäßig verteilt, was als Ausnahme von der Regel gewertet wird.

Mit diesen Daten wird deutlich, dass sich die Studie von Stratmann und Baur auf einen Zeitraum bezieht, in dem es besonders viele Direktabgeordnete in Wahlkreisausschüssen gegeben hat. So sind von der 12. bis zur 14. Wahlperiode Extrempunkte in beiden Verteilungen zu sehen.

\section{Rolle des Wahlsystems}

Angesichts der hier vorgelegten Befunde zu den Ausschussmitgliedschaften der Bundestagsabgeordneten von 1949 bis 2005 scheint die Rolle des Wahlsystems von Stratmann und Baur (wie auch von Medien) überbewertet worden zu sein. Kritisch muss ihre nicht näher begründete Auswahl des Zeitraums für die Untersuchung gesehen werden, denn in der 12 . bis 14. Wahlperiode - wie der Blick auf den gesamten Zeitraum von 1949 bis 2005 gezeigt hat - gab es eine außergewöhnliche Verteilung von Direktabgeordneten auf Wahlkreisausschüsse und Listenabgeordneten auf Parteiausschüsse. In der 1. und der 11. Wahlperiode waren in etwa gleich viele Direkt- und Listenabgeordnete in Parteiausschüssen vertreten. Und in der Zeit zwischen der 1. und der 11. Wahlperiode sind die Unterschiede zwischen den Mandatstypen in den Parteiausschüssen keineswegs derart deutlich, wie dies zwischen der 12. und der 14. Wahlperiode der Fall war. Erhärtet werden die Hinweise auf eine überschätzte Rolle des Wahlsystems durch die komplementären Befunde zu den Wahlkreisausschüssen. Bis auf die 1. Wahlperiode zeigen sich auch dort die Unterschiede zwischen Direkt- und Listenabgeordneten in der Zeit vor der 12. Wahlperiode keineswegs so deutlich, wie dies die Studie für die Zeit ausweist. In der 10. Wahlperiode gab es eine etwa gleiche 
Anzahl von Direkt- und Listenabgeordneten und in der 3. Wahlperiode sogar mehr Listenabgeordnete in Wahlkreisausschüssen.

Selbst wenn man Wahlkreis- und Parteiausschüsse als deutlich unterscheidbare Typen annimmt, wäre das Wahlsystem zur Erklärung der Allokation von Ausschussmitgliedschaften bestenfalls in einem Drittel der Fälle geeignet. Für weitere Forschungsarbeiten heißt das, dass entweder weitere Ausschüsse zu klassifizieren wären, um die empirische Basis zu erweitern. Oder theoretische Erklärungen der Verteilung von Ausschusssitzen müssen nach anderen Faktoren suchen.

\title{
Spenden an Abgeordnete
}

\author{
Philipp Austermann*
}

Die finanziellen Zuwendungen an Parteien und Politiker stehen stets unter der besonderen Beobachtung der Öffentlichkeit, wo die rechtlichen Rahmenbedingungen oftmals unklar sind. Daher werden im Folgenden die rechtlichen Grundlagen und Folgen finanzieller Zuwendungen an Abgeordnete des Deutschen Bundestages erläutert. ${ }^{1}$

\section{Zulässigkeit von Spenden an Mitglieder des Deutschen Bundestages}

Bevor die Zulässigkeit einer Spende an einen Bundestagsabgeordneten und etwaige Annahmeverbote geprüft werden können, ist die Spende zunächst von der Parteispende und von der unzulässigen Zuwendung abzugrenzen.

\subsection{Abgrenzung zur Parteispende}

Parteispenden sind nach $\$ 27$ Abs. 1 Satz 3 Parteiengesetz (PartG) alle über Mitglieds- und Mandatsträgerbeiträge hinausgehenden Zahlungen an politische Parteien im Sinne des $\$ 2$ PartG. Gemäß \$27 Abs. 1 Satz 4 PartG fallen unter den Spendenbegriff Sonderumlagen und Sammlungen sowie geldwerte Zuwendungen aller Art, sofern sie nicht üblicherweise unentgeltlich von Mitgliedern außerhalb eines Geschäftsbetriebes zur Verfügung gestellt werden. Parteispenden können auch von einem Abgeordneten stammen oder einem Abgeordneten übergeben werden, damit dieser sie bestimmungsgemäß an seine Partei weiterleitet. Nur Parteispenden sind gemäß $\$ 24$ Abs. 4 PartG im Rechenschaftsbericht der Partei aufzuführen.

* Der Autor, Angehöriger der Verwaltung des Deutschen Bundestages, gibt in dem Beitrag ausschließlich seine persönliche Meinung wieder.

1 Für die Abgeordneten der Landesparlamente gelten in der Regel ähnliche Vorschriften, zum Beispiel $₫ 4$ b Abs. 2 Nr. 2 Hessisches AbgG; $\$ 16$ Abs. 3 AbgG NRW. 